\section{Epidémiologie de l'accident vasculaire cérébral}

Groupe suisse de travail pour les maladies cérébrovasculaires et Fondation suisse de cardiologie ${ }^{1}$
Objectifs

1. Définition de l'accident vasculaire cérébral (nomenclature de la WHO)

2. Incidence et prévalence de l'AVC

3. Conséquences épidémiologiques les plus importantes de l'AVC

\begin{abstract}
Résumé
L'accident vasculaire cérébral (AVC) représente la troisième cause de décès dans les pays industrialisés après les maladies cardiovasculaires et les cancers. Il représente la cause la plus fréquente d'handicaps acquis à l'âge adulte. Pour ces raisons le traitement et la prévention sont d'une importance primordiale. Après la première manifestation de symptômes focaux d'ischémie cérébrale la probabilité de réapparition de symptômes à 5 ans est de $30 \%$, le risque étant avec $12 \%$ le plus élevé pendant la première année. L'incidence est estimée à 150 cas ou plus pour 100000 habitants par an.
\end{abstract}

\section{Tableau 1}

Comparaison et changements de la mortalité due à l'accident vasculaire cérébral chez les personnes âgées entre 40 et 69 ans, entre 1970 et 1985 [3].

\begin{tabular}{llll}
\hline Pays & $\begin{array}{l}\text { Hommes mortalité } \\
\text { pour 100 000 } \\
\text { habitants }\end{array}$ & $\begin{array}{l}\text { Femmes mortalité } \\
\text { pour 100 000 } \\
\text { habitants }\end{array}$ & $\begin{array}{l}\text { Changements de la } \\
\text { mortalité en \% entre } \\
1970 \text { et 1985 (hommes) }\end{array}$ \\
\hline Bulgarie & 249,2 & 155,8 & $+2,2$ \\
\hline Hongrie & 229,4 & 130,4 & $+3,9$ \\
\hline Roumanie & 176,6 & 129,2 & $+0,6$ \\
\hline Japon & 106,9 & 60,4 & $-7,1$ \\
\hline Ecosse & 99,3 & 77,0 & $-3,1$ \\
\hline Finlande & 98,1 & 57,3 & $-3,6$ \\
\hline Angleterre & 70,6 & 54,2 & $-3,3$ \\
\hline Allemagne & 68,2 & 38,8 & $-3,3$ \\
\hline France & 60,4 & 28,1 & $-4,5$ \\
\hline Norvège & 54,8 & 34,6 & $-3,5$ \\
\hline Suède & 48,1 & 30,5 & $-2,7$ \\
\hline USA & 45,4 & 35,1 & $-5,7$ \\
\hline Canada & 39,1 & 28,3 & $-4,6$ \\
\hline Suisse & 37,8 & 20,6 & \\
\hline & & & $-4,1$ \\
\hline
\end{tabular}

\section{Correspondance:}

PD Dr Philippe A. Lyrer

Clinique universitaire de neurologie

Hôpital cantonal

CH-4031 Bâle

e-mail: lyrerp@uhbs.ch

\section{Définition}

L'accident vasculaire cérébral se définit comme un trouble focal aigu du système nerveux central d'origine vasculaire. Environ 85\% des AVC sont d'origine ischémique et $15 \%$ d'origine hémorragique. Si les symptômes durent moins de 24 heures, on parle d'une attaque ischémique transitoire (AIT), s'ils persistent plus de 24 heures d'un accident vasculaire cérébral ou d'une apoplexie (en anglais: stroke) [1].

\section{Incidence et prévalence}

L'accident vasculaire cérébral représente la troisième cause de décès après les maladies cardiovasculaires et les cancers dans les pays industrialisés [2]. L'incidence s'élève à environ 150 cas pour 100000 habitants par an. La mortalité varie de pays en pays avec une marge de 20 à 250 décès pour 100000 habitants par an. En Suisse la mortalité pour tous les groupes d'âge était en 1985 de 20/100 000 (femmes) respectivement de 37/100000 (hommes) et représente ainsi le taux mondial le plus bas. En contrepartie la mortalité en Bulgarie était dans le même laps de temps de 249/100 000 pour les hommes et ainsi la plus élevée [3]. A l'intérieur de l'Europe la mortalité due à l'AVC est la plus basse dans les pays scandinaves, aux Pays-Bas et en Suisse et la plus élevée en Europe de l'Est (tabl. 1) [3].

Les chiffres cités se basent sur les statistiques de mortalité de la WHO d'après les données codées selon ICD-8 et -9. Cependant ces chiffres ne permettent pas de tirer de conclusions quant à l'étiologie de chaque

\footnotetext{
1 Les personnes suivantes ont participé par une contribution grande ou petite à la rédaction des articles sans avoir reçu la moindre indemnité financière:

M. Arnold, Berne; A. Barth, Berne; C. Bassetti, Berne; R. Baumgartner, Zurich; S. Beer, Valens; J. Bogousslavsky, Lausanne; A. Carota, Lausanne; A. Carruzzo, Lausanne; G. Devuyst, Lausanne; A. Dupont, Bâle; S. Engelter, Bâle; P. Erne, Lucerne; F. Fluri, Bâle; R. Guzman, Berne; S. Heffet, Genève; HJ. Hungerbühler, Aarau; HG. Imhof, Zurich; E. Keller, Zurich; H. Keller, Zurich; J. Kesselring, Valens; J. Le Floch-Rohr, Genève; K. Lövblad, Berne; Ph. Lyrer, Bâle (rédacteur de l'article); F. Mahler, Berne; A. Marx, Lugano; H. Mattle, Berne; B. Meier, Berne; R. Mordasini, Berne; M. Mosso, Zurich; F. Müller, Scherzingen; S. Osswald, Bâle; M. Reichhart, Lausanne; L. Remonda, Berne; D. Rüfenacht, Genève; W. Rutishauser, Genève; R. Sztaijzl, Genève; L. Regli, Lausanne; J. Rem, Bâle; A. Rivoir, Berne; G. Schroth, Berne; C. Städler, Lugano; P. Stierli, Aarau; M. Sturzenegger, Berne; B. Tettenborn, St-Gall; C. Tosi, Lugano; B. Weder, St-Gall; S. Windecker, Berne.
} 


\section{Figure 1}

Incidence de l'accident vasculaire cérébral dans une population définie à Framingham USA [6].

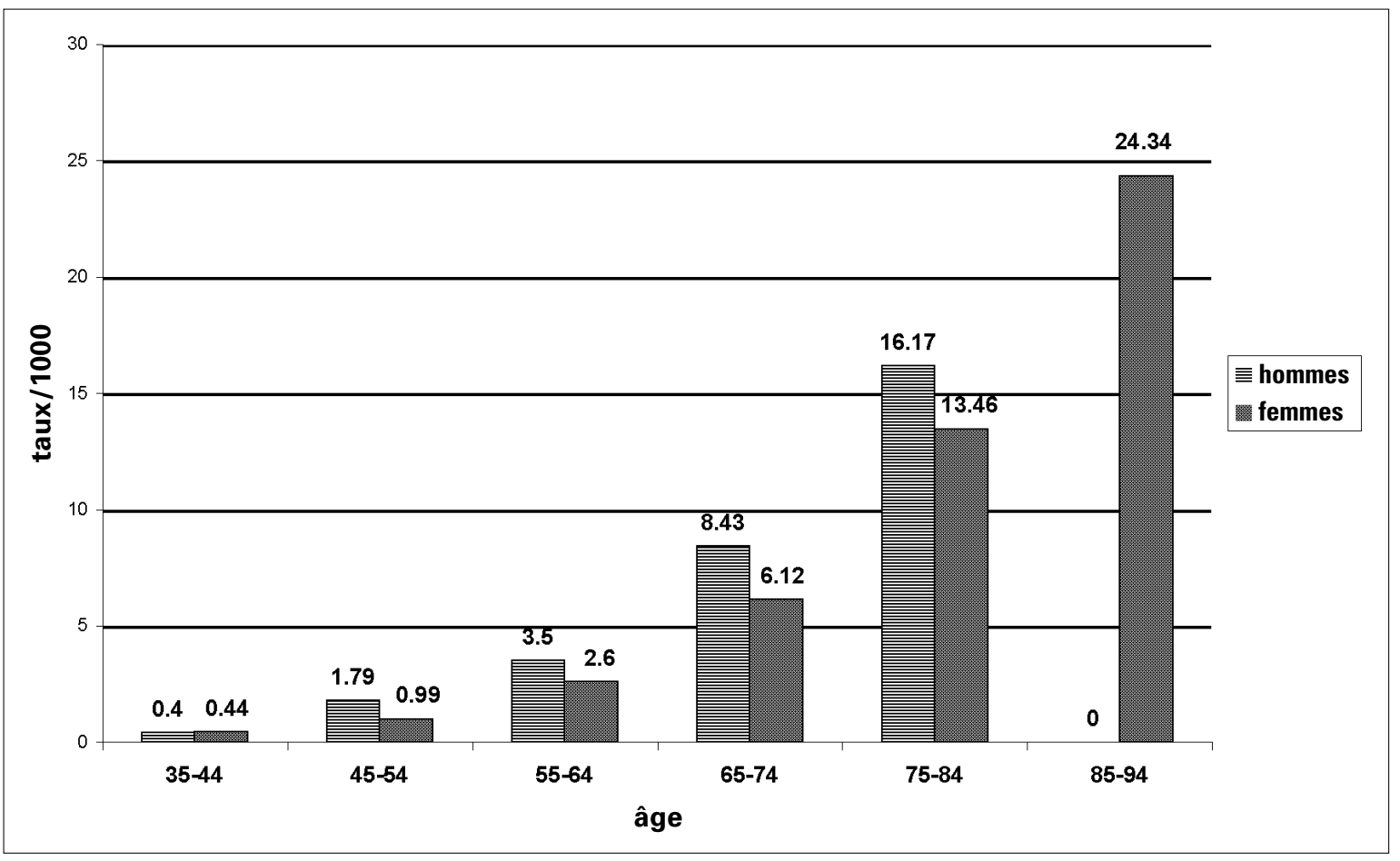

accident vasculaire cérébral en particulier. Le tableau 1 montre également que la mortalité dans de nombreux pays va en décroissant. Aux USA on a pu montrer entre 1969 et 1987 une baisse de 40\% chez les hommes et de $45 \%$ chez les femmes de la mortalité due aux accidents vasculaires cérébraux [3]. Différents facteurs peuvent être à l'origine de la diminution de la mortalité due à l'AVC: l'introduction de traitements efficaces contre l'hypertension artérielle, un meilleur traitement de l'accident vasculaire cérébral et de ses complications améliorant ainsi les chances de survie, un diagnostic posé plus fréquemment et précocement permettant l'introduction d'une prophylaxie secondaire. Avec l'amélioration des moyens diagnostiques il est d'ailleurs peu probable que le diagnostic d'AVC soit moins souvent posé $[3,4]$.

Dans le projet «Monitoring Trends and Determinants in Cardiovascular Disease (MONICA)" de la WHO qui se base sur la morbidité dans 27 pays, l'incidence d'accidents vasculaires cérébraux était également plus élevée chez les hommes que chez les femmes. La population examinée était de 2,9 millions de personnes. Dans la classe d'âge comprise entre 35 et 64 ans, l'incidence chez les hommes était de 141 à $344 / 100000$ et chez les femmes de 61 à 294. Pour les deux sexes on constate une augmentation de l'incidence avec l'âge, cependant moindre chez les femmes ce qui expliquerait en partie les différences entre les sexes dans la population mentionnée ci-dessus [5]. Le même phénomène a pu être observé dans l'étude de
Framingham citée plus haut. La figure 1 montre la relation entre l'incidence de l'AVC chez les femmes et chez les hommes [6]. Les figures 2a-c comparent l'incidence de l'AVC dans différents pays, séparés selon la tranche âge et le sexe [5]. On constate de grandes différences internationales de l'incidence pour tous les groupes d'âges. Les pays d'Europe de l'Est et quelques pays d'Europe du Nord présentent la plus haute incidence d'AVC: la Finlande, l'ex-Yougoslavie, la Russie et la Pologne. Dans les autres pays européens l'incidence diffère peu les uns des autres [5]. Différents taux d'incidence sont également constatés aux USA. Ainsi les Etats du sud-est ont les taux d'incidence les plus hauts alors qu'ils sont les plus bas dans les Etats montagnards de l'ouest. Une population composée de différents groupes ethniques pourrait être une des explications quant à ces grandes différences régionales aux USA. Ainsi les noirs auraient une plus grande quote-part d'apoplexies que les blancs [7]. Cela se traduit surtout par l'utilisation du système Medicaire qui rend compte de ces différences et qui montre le taux d'hospitalisation le plus élevé dans les Etats du sud-est [8].

Une étude épidémiologique publiée récemment en Allemagne montra dans une étude de cohorte s'appuyant sur l'ensemble de la population que l'incidence pour un premier AVC était de 2,0/1000 pour les hommes et de 1,7/1000 pour les femmes. La mortalité à 28 jours se montait à 19,4\% pour les AVC toutes causes confondues, elle fut la plus basse pour les AVC d'origine ischémique avec 11,5\% [9]. 


\section{Figure 2a}

Incidence de l'accident vasculaire cérébral dans le projet WHO Monica, comparaison entre différents pays en Europe dans la classe d'âge comprise entre 35 et 44 ans [4].

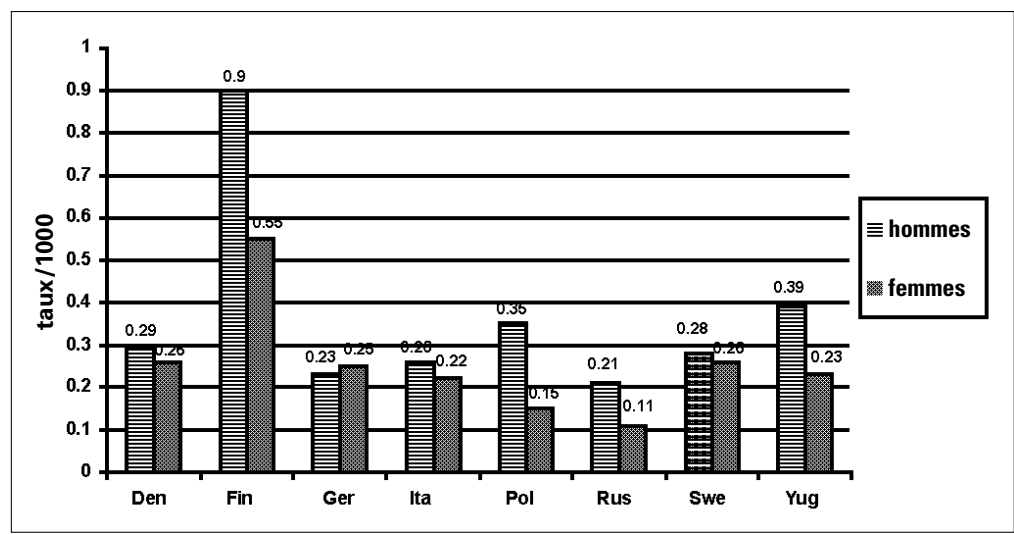

Figure $2 b$

Incidence de l'accident vasculaire cérébral dans le projet WHO Monica, comparaison entre différents pays en Europe dans la classe d'âge comprise entre 45 et 54 ans [4].

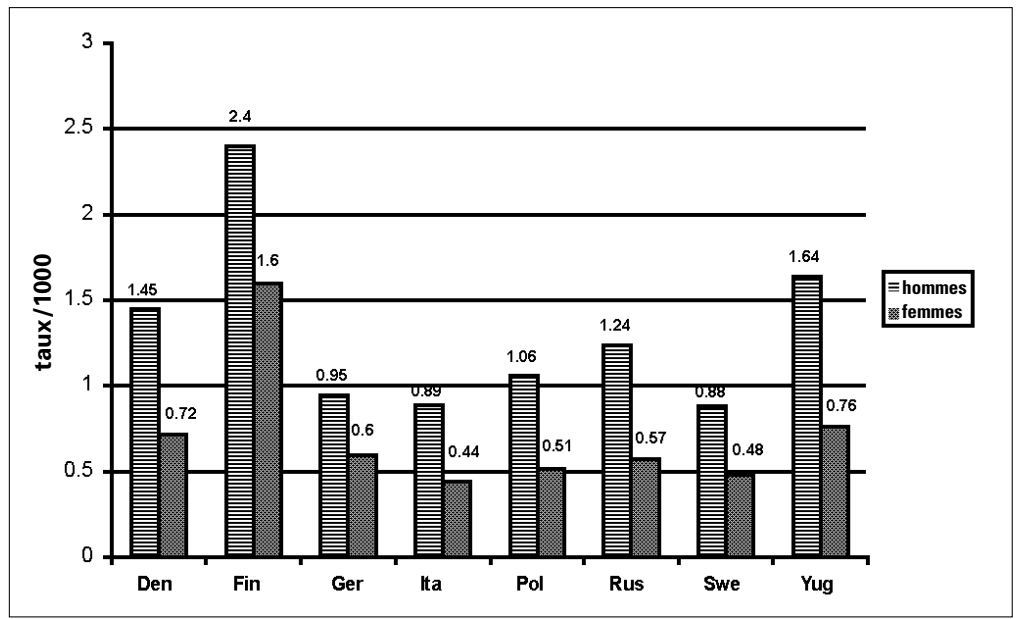

\section{Figure 2c}

Incidence de l'accident vasculaire cérébral dans le projet WHO Monica, comparaison entre différents pays en Europe dans la classe d'âge comprise entre 55 et 64 ans [4].

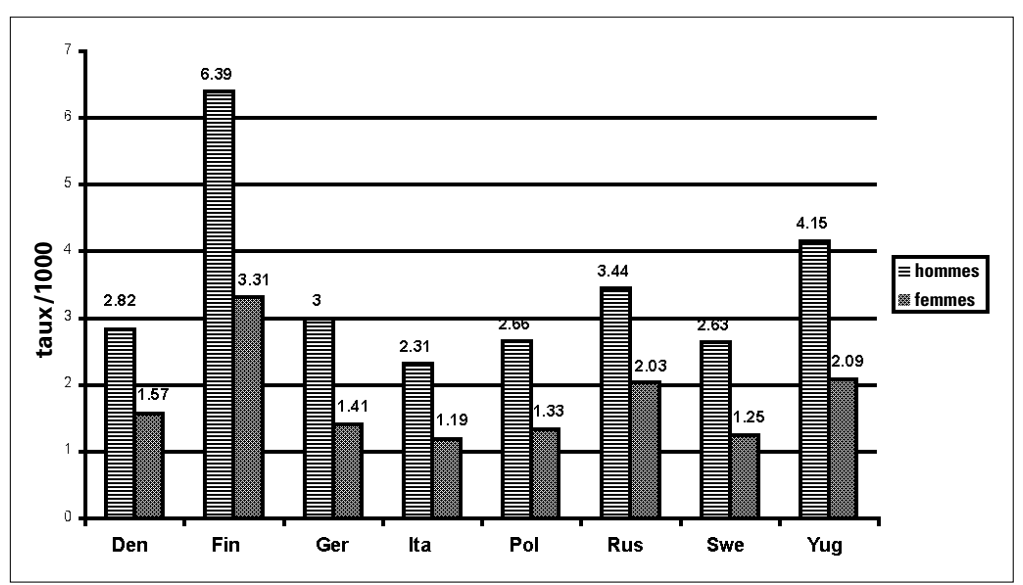

\section{Conséquences et coûts}

La charge financière que représentent les AVC pour le système de santé est énorme. Ils sont la cause la plus fréquente de handicaps acquis à l'âge adulte. Différents facteurs influent sur le handicap causé par l'AVC. Deux semaines après l'épisode aigu 60\% des patients ont besoin d'aide pour les activités de tous les jours, 70 à $80 \%$ sont handicapés pour la marche, 20 à $35 \%$ sont aphasiques, 60 à $70 \%$ ont des troubles de la vue. Il est difficile de faire une estimation des frais engendrés par un AVC. Ce n'est pas l'événement aigu à lui tout seul qui cause des frais, mais bien plus les moyens utilisés avant cet état pour diagnostiquer une maladie vasculaire, une autre maladie ou dans l'idée d'introduire un traitement préventif. En Suède, pour l'année 1991 une somme de 232,4 millions US\$ / 1000000 habitants a été calculée [10]. Ne sont pas inclus dans ces chiffres les frais occasionnés par la réhabilitation, respectivement les frais pour les handicaps restant.

Les chiffres actuels pour la Suisse ont été disponibles grâce à la participation de plusieurs cliniques au "International Stroke Trial» (IST). Ces chiffres ont été rassemblés de mars 1993 jusqu'en mai 1996 $[6,10,11]$. L'objectif de l'étude était de démontrer l'efficacité de l'acide acétylsalicylique et de l'héparine dans le traitement aigu de l'AVC. 28\% des patients suisses avaient un gros infarctus dans le territoire carotidien, 39\% des infarctus partiels dans le territoire carotidien, 16\% des infarctus dans le territoire vertébrobasilaire et $16 \%$ avaient un infarctus lacunaire. Chez 74\% des patients fut effectué un scanner cérébral avant la randomisation. Lors du contrôle ou du questionnaire ultérieur des patients traités en Suisse on a pu constater qu'après 2 semaines $52 \%$ des patients avaient pu regagner leur domicile et qu'après 6 mois, $77 \%$ des patients étaient encore en vie. Parmi ces derniers, $69 \%$ vivaient à la maison, $17 \%$ dans des institutions spécialisées, $6 \%$ dans un autre hôpital et 6\% dans un asile de vieillards. Le pourcentage de patients placés dans des institutions spécialisées était le double par rapport aux données moyennes de l'étude [12]. On pense que le pourcentage élevé de gros infarctus dans le territoire carotidien mène à un besoin accru de soins spécialisés des patients suisses en comparaison avec les patients des autres pays. Les chiffres publiés par les «Stroke Units» ont montrés qu'environ $10 \%$ des patients hospitalisés pour un AVC avaient un gros infarctus dans le territoire carotidien [11, 13]. Ainsi la proportion de patients qui restent au bout du compte dépendants de soins serait inférieure aux chiffres mentionnés dans l'étude IST. Sur la base de ces chiffres il faut cependant compter par année rien que pour la Suisse entre 400 et 1200 patients dépendants de soins suite à un AVC. 


\section{Références}

1 WHO/MNH task force on stroke and other cerebrovascular disorders 1989. Stroke 1989. Recommendations on stroke prevention, diagnosis, and therapy. Report of the WHO Task Force on Stroke and other Cerebrovascular Disorders. Stroke 1989;20:1407-31.

2 Bogousslavsky J (Ed.). Stroke prevention by the practitioner. Karger, 1999.

3 Bonita R, Stewart A, Beaglehole R. International trends in stroke mortality: 1970-1985. Stroke 1990;21:989-92.

4 Thorvaldsen P, Kuulasmaa K, Rajakangas AM, Rastenyte D, Sarti C, Wilhelmsen L. Stroke trends in the WHO MONICA project. Stroke 1997;28:500-6.

5 Modan B, Wagener DK. Some epidemiological aspects of stroke: mortality/morbidity trends, age, sex, race, socioeconomic status. Stroke 1992;23:1230-6.

6 Wolf PA, D'Agostino RB. Epidemiology of stroke. In: Barnett HJM, Mohr JP, Stein BM, Yatsu FM (eds). Stroke. Pathophysiology, Diagnosis, and Management. 3rd ed. New York: Churchill Livingstone, 1998. p. 3-28.

7 Pickle LW, Mungiole M, Gillum RF. Geographic variation in stroke mortality in blacks and whites in the United States. Stroke 1997;28:1639-47.

8 Lanska DJ, Kryscio R. Geographic distribution of hospitalization rates, case fatality, and mortality from stroke in the United States. Neurology 1994;44:1541-50.
9 Asplund K, Marké L-A, Terént C, Wester P.0. Costs and gains in stroke prevention: European Perspective. Cerebrovasc Dis 1993;3(suppl 1):34-42.

10 International Stroke Trial Collaborative Group. The International Stroke Trial (IST): a randomised trial of aspirin, subcutaneous heparin, both, or neither among 19435 patients with acute ischaemic stroke. Lancet 1997;349:1569-81.

11 Lyrer P. Neue Ansätze in der Akutbehandlung des zerebrovaskulären Insultes. Schweiz Med Wochenschr 1994; 124:2005-12.

12 Coselli JS, LeMaire SA. Experience with retrograde cerebral perfusion during proximal aortic surgery in 290 patients. J Card Surg 1997;12:322-5.

13 Mattle HP, Eicher VE, Bassetti C, Sandercock P. International Stroke Trial Switzerland: some epidemiologic data. Schweiz Med Wochenschr 1999;129:1964-9.

Des tirés à part peuvent être obtenu dès l'automne 2000 auprès de la Fondation suisse de cardiologie, Schwarztorstrasse 14, Case postale, 3000 Berne 14.

Deutsch erschienen in Nr. 16/2000 